\title{
Zentralvorstandssitzungen im Sommer 2010
}

\begin{abstract}
Medical Board - Nach der Pilotphase tritt die FMH der Trägerschaft des von der GDK unterstützten nationalen Medical Board bei. Dabei geht es darum, die Entwicklung von HTA (Health Technology Assessment) in der Schweiz zu fördern.
\end{abstract}

Strategische Allianz «Gesundheitskompetenz»Die FMH tritt der Allianz bei, um deren Stossrichtung mitzubestimmen; dies auch im Hinblick darauf, dass Gesundheitskompetenz im neuen Präventionsgesetz prioritär ist.

Basis-Qualitätsmodell - Der ZV unterstützt dieses Pilotprojekt des VEDAG mittels einer Aufwandentschädigung für teilnehmende Ärztinnen und Ärzte.

«Strategie eHealth Schweiz» - Anlässlich des Hearings zum rechtlichen Regelungsbedarf fokussierte die Ressortleitung auf das elektronische Patientendossier und dessen Primärzweck, die Unterstützung und Optimierung der Patientenbehandlung. Ebenso empfahl sie, die doppelte Freiwilligkeit von Patienten und Ärzten konsequent einzuhalten.

Tarifrevision TARMED FMH - Die FMH führt mit $\mathrm{H}+$ die Revision der Tarifstruktur TARMED eigenständig durch und schafft die notwendigen personellen Ressourcen dafür.

FUTURO/Chronic Care - Der ZV lässt sich über das Projekt FUTURO zur Abgeltung nichtärztlicher Leistung im Chronic-Care-Bereich informieren; Voraussetzung dafür wird ein Fachausweis in «Chronic Care» für die Medizinischen Praxisassistentinnen sein.

Seminar «Medizin und Ökonomie» - Der Zentralvorstand der FMH vergibt das FMH-Logo für den Flyer des neuen Seminars «Medizin und Ökonomie», eines gemeinsamen Angebots von FMH und $\mathrm{H}+$.
Dachverband Ipsilon - Der ZV will Ipsilon weiter fördern, damit die «Initiative zur Prävention von Suizid in der Schweiz» Bekanntheit gewinnt und u.a. sowohl psychiatrische als auch sozialwissenschaftliche Forschung betreiben kann.

Übergriffe in Arztpraxen - Die FMH bildet eine Arbeitsgruppe, die sich mit dem Thema auseinandersetzt und Massnahmen vorschlägt, wie das Standesverfahren wirkungsvoller gestaltet und die Zusammenarbeit mit den Aufsichtsbehörden verbessert werden kann.

DRG-Begleitforschung - Die FMH schreibt das Thema «Entwicklung der Rahmenbedingungen für Spitalärzte und der Arzt-Patienten-Beziehung» für die DRG-Begleitforschung aus. Zudem erhebt sie in der Assistentenumfrage 2010 des SIWF Daten zum Dokumentationsaufwand und dem Wissensstand zu SwissDRG.

Telefoneintrag für Telemedizinanbieter - Telemedizinanbieter sollen im Telefonbuch künftig nicht mehr unter der Rubrik «Ärzte» erscheinen; die FMH wird deshalb eine neue Rubrik initiieren.

Integrierte Medikamentenverordnung - Zur Klärung der Termini dieses Bereiches, insbesondere des eRezepts, hat der ZV ein Grundlagenpapier erstellen lassen; Teil davon sind auch Empfehlungen, u. a. zur Förderung der integrierten Medikamentenversorgung. 